\title{
Mechanisms of change in brief treatments for borderline personality disorder: a randomized controlled trial
}

Ueli Kramer ( $\boldsymbol{\nabla}$ ueli.kramer@chuv.ch )

Institute of Psychotherapy, University of Lausanne

Loris Grandjean

University Clinic Lausanne

Hélène Beuchat

University Clinic Lausanne

Stéphane Kolly

University Clinic Lausanne

Philippe Conus

University Clinic Lausanne

Yves de Roten

University Clinic Lausanne

\section{Bogdan Draganski}

University Clinic Lausanne

Jean-Nicolas Despland

University Clinic Lausanne

\section{Study protocol}

Keywords: Psychiatric Treatment, Mechanisms of Change, Borderline Personality Disorder, Process, Emotional Processing, Theory of Mind Processing, fMRI

Posted Date: January 24th, 2020

DOl: https://doi.org/10.21203/rs.2.21827/v1

License: (c) (i) This work is licensed under a Creative Commons Attribution 4.0 International License. Read Full License

Version of Record: A version of this preprint was published at Trials on April 16th, 2020. See the published version at https://doi.org/10.1186/s13063-020-4229-z. 


\section{Abstract}

\section{Background}

Borderline Personality Disorder (BPD) is one of the most frequent, most debilitating and lethal mental conditions and is associated with a serious burden of disease. Treatment for patients with BPD involves structured psychotherapy, and may involve brief psychiatric treatment as first-line intervention. No controlled study has assessed the effectiveness of such brief intervention. Whereas most psychotherapy studies with patients with BPD focus on the effectiveness of the intervention, we still lack an understanding of how and why these effects are produced from a patient process perspective. It is therefore of utmost importance to study the treatment-underlying mechanisms of change. The present study plans to apply novel measurement methods for assessing change in two central psychobiological processes in BPD: emotion and socio-cognitive processing. The study uses theory-driven and ecologically valid experimental tasks which take as anchor the patient's individual experience, by integrating methodology from psychotherapy process and neurofunctional imagery research.

Methods and study design

The present two-arm randomized controlled study aims at testing the effects (i.e., symptom reduction) and the underlying mechanisms of change associated with a brief psychiatric treatment (10 sessions over 4 months), compared with treatment as usual. Participants ( $N=80$ patients with BPD) undergo assessments at four points (intake, 2 months, discharge, and 12 month follow-up). In addition to symptom measures, individuals undergo a two-step assessment for the potential mechanisms of change (i.e., emotion and socio-cognitive processing): a) behavioural and b) (for a sub-sample) neurofunctional. We hypothesize that change in the mechanisms explains the treatment effects.

\section{Discussion}

The present study uses an easy-to-implement treatment of BPD, as well as a sophisticated assessment procedure to demonstrate the critical role of psychobiological change in emotion and socio-cognitive processing in brief treatments. It will help increase the effectiveness of brief treatment for BPD and help diminish the societal burden of disease related with BPD, in these early stages of treatment.

Trial registration : Clinical Trials NCT0317818 (date of registration of Abstract October 24 th , 2018). Protocol version number 2 from February 9 th , 2018.

\section{Administrative Information}


Title (1) Mechanisms of change in brief treatments for borderline personality disorder: a randomized controlled trial

Trial registration Clinical Trials NCT03717818

$(2 \mathrm{a} / \mathrm{b})$

Protocol version Number 2 from February $9^{\text {th }}, 2018$

(3)

Funding (4) This trial is supported by the Swiss National Science Foundation

(SNSF100014_179457/1, to Dr. Kramer).

Author details Ueli Kramer, Loris Grandjean, Hélène Beuchat, Stéphane Kolly, Philippe Conus,

(5a) Yves de Roten, Bogdan Draganski, Jean-Nicolas Despland

Name and PD Dr. Ueli Kramer: Veli.Kramer@chuv.ch

contact

information (5b)

Role of sponsor The Swiss National Science Foundation is funding this project, but does not $(5 c)$

have any influence on any level of content or procedure of this project.

\section{Background}

Borderline Personality Disorder (BPD) is one of the most prevalent mental disorders with $2-3 \%$ in the general population. Direct societal costs are related with frequent emergency service use, intense use of inpatient and outpatient treatments, indirect societal costs stemming from prolonged sick leaves, abusive street drug consumption, intra-familiar abuse and neglect and in some cases legal costs $(1,2)$.

Psychological treatments are considered first-line for problems related with Personality Disorders (3-6). Whereas there are several theoretical accounts on how these effects are produced, there is a paucity of systematic empirical research focusing on mechanisms of change in treatments of PDs (7-13), aiming at empirically explaining how treatments work and, ultimately, increasing the treatment's effectiveness. Such an empirical understanding would be particularly useful at the beginning of treatment: a better knowledge of the determinants of initial symptom alleviation in psychological treatment would allow to deliver even more potent treatments for these patients from the very first session on, and to prevent some of the long-term consequences of the disorder.

The objective of the present study is to explain early symptom change in patients with borderline personality disorder undergoing a brief psychiatric treatment that is consistent with the international and national treatment guidelines $(3,14)$. We assume that symptom change is the result of a complex interplay between changing central process characteristics of the patient - assessed from an integrated neuro-behavioral perspective - and the moment-by-moment responsive adjustment by the therapist to them. The present trial examines two patient-related mechanisms of change in treatments of BPD: (1) emotion processing (2) change in socio-cognitive processing. By aiming at discovering the underlying "laws of change" in patients undergoing brief treatments for BPD, such research may help increase the 
effectiveness of any bona fide therapy approach from the very first session on and thus may help decrease direct and indirect societal costs related with BPD.

In order to achieve this, we aim at studying a specific brief guideline-based intervention for BPD. In addition to the structured psychotherapy models validated for the treatment of Borderline Personality Disorder (e.g., Dialectical-Behavior Therapy, Mentalization-Based Therapy, Transference-Focused Psychotherapy), the past 10 years have witnessed the emergence of psychiatric approaches to the treatment of BPD $(15,16)$. In a randomized controlled trial, McMain and colleagues $(17,18)$ have tested the efficacy of a 1-year long Dialectical-Behavior treatment program to an equally long General Psychiatric Management GPM $(19,20)$. The results were somewhat unexpected, showing that both treatments did equally well on all outcome indices and most process characteristics, including symptom relief until 2 years after the end of treatment. Bateman and Fonagy (2009) used a similar type of psychiatric approach in which they found consistent results with regard to the comparison with mentalization-based treatment (MBT) (21). These emerging studies may suggest that for at least the first phase of treatment of patients with BPD, it might be sufficient to offer a psychiatric intervention which is easily learnable; as such, we increase general access to mental health intervention for a large amount of patients with BPD $(22,23)$. Such new health care models have been advocated within the stepped-care literature for BPD (24-26).

To date, no study has examined such a brief guideline-based psychiatric intervention, together with its explanatory mechanisms of change for outcome, compared to a treatment as usual. This is the aim of the present research. It will determine the effectiveness of a brief psychiatric intervention and detail its psychobiological underpinnings of change.

A methodological problem and a possible solution

Earlier studies focusing on mechanisms of change in treatments for BPD fall into two major categories, neglecting a possible methodological integration explaining psychobiological change. Some studies used psychotherapy process research, describing change on patient central variables in-session (27-29) favoring external clinical validity, but remaining sensitive to the session-internal influences by the patienttherapist interaction (i.e., therapist responsiveness; (30). Other studies used biological assessments of change where the changing variable was observed on neurobiological activation to the exposed standardized stimuli $(31,32)$ - favoring internal validity, but neglecting the idiographic content so central to understand change in psychotherapy.

In order to address these methodological problems, we suggest researchers should carefully integrate methods from psychotherapy process research with neurofunctional methods, by taking into account the individual's subjective experience as anchor - substantiated in the form of individualized stimuli in the experiment - in the assessment of the mechanisms $(12,33)$. This is only meaningful when the design controls for a number of manipulation checks (see under Methods). Such an integrated experimental design goes beyond the systematic assessment of the therapy process, as described by our earlier studies $(28,34,35)$, in addition, it becomes possible to relate aspects of the patient's subjective 
experience to the neurobehavioral correlates of change (see also the discussion by Tashiro et al., 2006, and Kramer, $2019(12,36))$.

Mechanisms of change in treatments for borderline personality disorder

Here below, we will focus on two potential mechanisms of change which are among the most potent so far, and embed them within our integrative conception of mechanisms of change: (1) emotion processing, (2) socio-cognitive processing.

Emotion processing: change in affect-meaning states related to self-criticism

Change in emotion processing is central for psychotherapy in patients with BPD (37). Greenberg and colleague (2006) have differentiated between, among others, emotion regulation and emotion transformation (38). With regard to emotion regulation over the course of psychotherapy, Neacsiu and colleagues (2010) showed that the acquisition of specific coping skills strategies, aiming at more efficient emotion regulation functioned as mediator of change in DBT (39). These findings were in line with results found in a pilot fMRI study: Schnell and colleague (2007) investigated cognitive reappraisal over the course of inpatient DBT for $\mathrm{N}=6$ patients with BPD (31). The authors showed a decrease in activation over the course of therapy on the levels of the right ACC, the temporal and posterior cingulated cortex and the left insula (31). Using a previously validated procedure of cognitive reappraisal (40), Schmitt and colleagues (2016) confirmed parts of these results on a sample of patients with BPD ( $N=32$ patients) undergoing DBT and showed an enhanced neural connectivity between ventro-lateral pre-frontal cortex areas and the amygdala (32), consistent with the neuropathological model put forward by New and colleagues (2007)(41). Comparable results were obtained on $N=11$ patients with BPD undergoing DBT, compared with $N=11$ healthy controls: a decreased amygdalar reactivity was found after DBT $(42,43)$. Consistent results were found for change in distraction as emotion regulation strategy (44) and change in pain-mediated emotion regulation (45). Perez and colleagues (2015) showed consistent effects speaking in favor of enhanced fronto-limbic connectivity after a one-year long Transference-Focused Psychotherapy (TFP) for $\mathrm{N}=10$ patients with BPD (46). Taken together, these results suggest improved emotion regulation capacities after therapy for patients with BPD, but more research integrated within a larger theoretical framework is necessary.

In addition to the regulation perspective on emotion, we define emotion transformation as the change process by which emotions unfold and change over time from the least productive to the most productive, the latter being underpinned my increased meaning making (47). It was shown that this process relates to healthy functioning and good psychotherapy-outcome (34), in particular the flexibility of emotional experiences (47). The relevance of this dynamic conception of emotion processing for BPD was supported in terms of change in anger processing (48) and in terms of change in undifferentiated global distress (27). It was shown to also be of relevance in narcissistic and histrionic PDs $(49,51)$.

This transformation conception may explain the resolution of harsh self-criticism, a central clinical feature of BPD $(48,52-54)$. Whelton and Greenberg (2005) proposed a paradigm of studying emotion 
transformation related to self-criticism using the empty chair dialogue. Patients criticized themselves in a structured assessment procedure using imaginative and emotion-eliciting enactment tasks. It appeared that the depressive persons presented more self-contemptuousness in their self-criticism, compared to controls and presented with higher levels of shame, sadness and emotional collapse, along with less pride (55). This study was on $\mathrm{N}=45$ undergraduates presenting with and without anger problems, using the same paradigm (49). What differentiated the two groups was the presence of self-contemptuousness, $t(1,43)=1.91, p<.05)$ associated with the self-criticism, along with the absence of the existential need in the anger-prone individuals. This means that for anger-prone participants - which share this clinical feature to some extent with BPD -, self-criticism is particularly harmful to the emotion transformation when associated with self-contemptuousness. We may therefore assume that decrease in selfcontemptuousness - and possibly increase in its antidote self-compassion - and increase in emotion flexibility are markers of productive change in treatment.

Emotion transformation related to self-criticism is underpinned by biological changes. Using standardized stimuli, Longe et al. (2010) showed in a female student sample ( $\mathrm{N}=17)$ a BOLD activation (intra-subject comparison to a neutral condition at the level $p<.05$ corrected) in the left pre-frontal cortex (PFC; BA 45), in the lateral orbito-frontal cortex (OFC; BA 47), in the left dorsolateral PFC (BA 9), inferior and middle temporal gyrus (BA $20 \& 21$, including lingual gyrus, BA 19) (56). The hyperactivity in pre- and orbito-frontal regions associated with self-criticism in this study was interpreted as linked with the inhibitory behavior known to be associated with activation of the lateral PFC (57). Brain activity in the striatum was associated with self-punishing emotions of self-criticism (55), such as shame, anger about the self and self-contemptuousness (35). In addition, some regions of the insula-basal ganglia networks are known to be associated with disgust processing (58). In an fMRI study using individualized selfcritical stimuli (which were previously selected based on a large set of words), Doerig and colleagues (2013) showed bilateral insula activation, along with activations in left hippocampus and amygdalar formations, interpreted as regions recruited in emotion processing of self-critical stimuli (59). More research is needed to understand change in self-contemptuousness and its neuronal substrates over the course of treatment for BPD, when an individualized measurement method is applied.

Change in socio-cognitive processing: integrating "hot" interpersonal information

Change in the patient's socio-cognitive, or mentalizing capacities is a discussed putative mechanism of change in the treatment of $\operatorname{BPD}(53,60,61)$. Levy and colleagues $(2006)$ examined change in three forms of psychotherapy for BPD - TFP, DBT and supportive therapy - and found that only TFP was associated with the increase of mentalizing functions, along with development of more secure attachment patterns for some patients in this group (29). Consistent results were presented by Fischer-Kern and colleagues (62) (see also de Meulemeester et al (63); Maillard et al., (64)). Other research has underlined the moderating factor of mentalizing capacities for outcome for different categories of PD $(65,66)$. To our knowledge, no studies have shown mediation for changes in socio-cognitive processing in treatments for BPD. 
One method to investigate the core interpersonal contents related with attachment figures (i.e., "hot" stimuli), again formulated from an individualized perspective, is the core conflictual relationship theme (CCRT;(67)). A CCRT is a formulation composed by a patient's wish (e.g., to be close, to be treated harshly), the anticipated response of the other/the object (e.g., to facilitate one's independence, to be harsh) and the response of the self (e.g., to feel understood, to be frustrated). According to Luborsky (1998), pervasiveness of a CCRT is the degree of generality of a theme across specific relationship episodes and specific interactions(67). In patients with BPD, one may identify a central theme for each person which is present in more than $60 \%$ of the specific relationship episodes $(68,69)$. After treatment, it is expected that pervasiveness related to the core theme decreases. Luborsky (1998) demonstrated for $\mathrm{N}$ = 33 patients undergoing psychodynamic psychotherapy - although not patients with BPD -, a pre-post decrease of pervasiveness over time $(F(1,32)=7.4, p<.01)$ which was particularly strong for the category of the negative response of the self. This decrease correlated with symptom change at the end of treatment (67). Therapy studies on patients with BPD are needed, to test the role of decrease in CCRT pervasiveness over time.

As such, an emerging field of research focuses on the explanatory roles of (a) the patient's emotion processing, (b) the patient's capacities of socio-cognitive processing, for treatment effects related with PDs. We think that the most promising assessments rely on an integration between idiographic and nomothetic assessment procedures.

Objectives and research hypotheses

1. (Outcome) A 10-session BPD-specific guideline-based treatment produces more reduction in specific borderline symptoms than a non-specific treatment as usual (TAU). 2. (Global change) A 10-session BPDspecific treatment presents pre-post change in socio-cognitive processing (SCP) and emotion processing (EP), which is not the case in the TAU. 3. (Treatment response) SCP and EP change more in treatment responders, compared to non-responders (across conditions). 4. (Mediation) The changes in these potential mechanisms of change will function as mediators of symptom decrease (between intake, discharge and follow-up; the latter will be used to disentangle potential time confounds). Change is operationalized on behavioral and neurofunctional (by controlling for the corresponding behavioral/idiographic information) levels. For post-therapy EP, we expect a greater emotional variability when dealing with individualized self-criticism, along with lesser self-contemptuousness, compared with pre. It is expected that post-therapy neuronal activations are lower in a EP network involving areas in the PFC, the striatum and the insula-basal ganglia $(58,59)$, when compared with pre. For post-therapy SCP, we expect a lower CCRT pervasiveness, when compared with pre, and we expect that post-therapy neurofunctional activations are lower in regions associated with the theory of mind (i.e., anterior cingulate, precuneus, inferior and middle frontal gyri and inferior parietal lobes; (70-72), compared with pre.

\section{Methods}


The present study is planned as a randomized controlled treatment trial (trial design ), involving a guideline-based psychiatric treatment (General Psychiatric Management; GPM; (20)) for BPD, over the course of a 4-month-plus-12-month-follow-up treatment program, in comparison with a 4-month treatment as usual, by focusing on the underlying mechanisms of change. The outcome part is a superiority trial, while the mechanisms part focuses on controlled comparison of processes of change. The trial takes place in the context of a University Hospital (study setting ).

\section{Ethical Clearance}

With respect to the submitted project on the SwissEthics plateform, the competent Canton de Vaud Ethics Committee, has approved the study (2017-02167). The study is registered (NCT0317818). In keeping with the established, and approved, Data Management Plan, only anonymous data will be kept in the file. All video raw data, where the patient may be identified, as well as fMRI data, will be stored separately from patient identifiers and from the main dataset.

Inclusion Criteria Of The Patients

Patients with Borderline Personality Disorder (BPD) according to Diagnostic and Statistical Manual of Mental Disorders, Fifth Edition (73) mastering French to a sufficient extent will be included. Rate of comorbidity is expected to be high. All patients accepting the study by informed written consent will be included in the outcome and mechanisms parts of the study; a sub-group of patients with additional inclusions criteria (female, younger than 45 years, right-handed, no or stable medication and absence of formal counter-indication on the security check) will be included in the fMRI part of the study.

\section{Exclusion Criteria Of The Patients}

Patients with neurocognitive disorder, psychosis and bipolar disorder I will be excluded from the trial. In order to ensure generalizability of the results to a wide variety of clinical settings, no other exclusion criteria will be applied.

Patients And Therapists

Based on a power-analysis (presumed power .819 , for 2 concomitant mechanisms, $d=0.60$; two-tailed alpha $=.05 ; 30 \%$ drop-out), $\mathrm{N}=80$ patients presenting with BPD are planned to be recruited for the present study at the Department of Psychiatry, University of Lausanne, Switzerland. In total, N = 10 therapists (psychiatrists or psychologists) participate in the study for the GPM treatment; they have at least 6 hours of specific training in psychiatric treatment for BPD (74), in addition to training in psychotherapy according to Federal regulations.

Randomization Procedures

Block randomization (blocks of 10) will be used throughout the study. The randomization will be performed online by an independent researcher, who prepares sealed enveloped informing about the 
attribution to one out of two conditions. Informed consent will be obtained by a trained researcher (informed consent ). Upon inclusion into the protocol, the envelope will be opened and the condition will be communicated to patient and therapist. The (outcome and mechanisms) assessors and data analysts will be blind to the condition, but the patients nor the therapist will not be. In order to control for biases, assessors will be polled at the end of the study with regard to each patient's condition . No a priori stratification will be performed.

Interventions

Brief treatment encompasses the communication about diagnoses, problem areas, anamnesis, the work on treatment focus, objectives and motivation, the treatment of treatment-interfering problems and the elaboration of interpretations related with the core concept of interpersonal hypersensitivity, according to the principles of General Psychiatric Management for BPD (GPM; $(20,75-77)$. Therapist adherence to the protocol will be self-assessed by therapist using Gunderson's (2016) questionnaire for adherence to good psychiatric management principles, to be filled in after each treatment . For the TAU, 10 therapists will intervene using non-specific crisis management as usual (minimal ethical assessment and contact with the patient according the local directives, ensuring safety management). Should a participant request discontinuation of the intervention, the study therapist will use this as criteria for discontinuing and modifying the allocated intervention. Relevant concomitant care was permitted during the trial, and was recorded.

Assessments

Assessments take place at intake, 2 months and discharge, plus at follow-up after 12 months. At followup, the participants will be contacted by the research team .

Instruments And Materials

The main outcome measure is the Zanarini Rating Scale for Borderline Personality Disorder (ZAN-BPD (78)). ZAN-BPD is a continuous hetero-administered measure assessing the nine criteria outlined in DSM5 , on a continuous Likert-type scale ranging from 0 to 4 . As such, it yields a total theoretical score of 36 . A comprehensive validation study has shown its reliability, validity and sensitivity to change (78).

Emotion processing related to self-criticism will be assessed using the self-criticism task. This task involves two main steps. (1) Conduct of a two-chair dialogue on self-criticism, an individualized and therefore particularly emotion-arousing procedure $((35,55,79)$ and observer's process rating of the patient's emotions using the Classification of Affective Meaning States (CAMS; $(80)$; see also $(34,47)$ ) with the aim of extracting $n=20$ individualized self-critical words for each patient at each assessment point. Increase in emotion flexibility (i.e., more different emotion categories as a reaction to the selfcriticism) over time, along with a decrease in self-contemptuousness associated with the self-criticism over time, are indicators of productive change. (2) Test, one week later, of neural correlates of the processing related to the $n=20$ individualized self-critical words (extracted from step 1 ), in comparison 
with a set of $\mathrm{n}=20$ negative emotional (81), $\mathrm{n}=20$ positive emotional (81), $\mathrm{n}=20$ neutral words and $\mathrm{n}=$ 20 non-words (symbols; in total $\mathrm{N}=100$ words; presented in a random order). Self-reported emotional arousal (on SAM) and self-esteem (on SSES), along with observer-reported fear/shame (on CAMS) reaction, will be measured as manipulation checks for step 1; SAM assessment will be done for each stimulus presented in the scanner. This task was empirically tested in a non-published pilot study: for $\mathrm{n}=$ 5 individuals, we showed for the individualized words higher subjective arousal levels than for the standardized negative words, along with comparable neurofunctional activations.

Change in socio-cognitive processing of interpersonal patterns will be assessed using two independent tasks, one behavioral, and the other fMRI. The behavioral task involves the conduct of a structured interview using the Relationship Anecdote Paradigm (67) and based on this video-taped structured material, observer's process rating of the patient narrative using the CCRT (67-69) with the aim of comparing its pervasiveness pre-post-follow-up (see Fig. 1). Decrease in CCRT pervasiveness is an indicator of productive change. The fMRI task involves the appreciation of humoristic stimuli measuring the patient's theory of mind; this task has previously been validated for BPD ((70); see for the validation of the stimuli $(82,83)$ ). It involves the processing and understanding of three sets of stimuli, presented in a pseudo-random order: (1) ToM (theory of mind): visual jokes requiring attributing false mental states to the protagonists presented in the cartoons (30 stimuli), (2) PUN (visual puns): visual puns, i.e., cartoons that are based on visual similarities, not requiring attributing false mental states (30 stimuli) and (3) a non-humorous control condition with incongruent visual information (30 stimuli, in total $\mathrm{N}=90$ ). Manipulation checks involve the assessment of the understanding of each joke. Decrease in activation of ToM network over time is an indicator of productive change.

The remaining assessments concern secondary outcomes and include the Outcome Questionnaire-45 (84) which is a self-report questionnaire comprising 45 items aiming at assessing psychotherapeutic results, including a global score and three sub-scale scores: symptomatic level, interpersonal relationships and social role. It has been translated and validated in French (85). The Borderline Symptom List (BSL-23, (86)) is a self-report questionnaire assessing the BPD symptomatology using 23 items ; it represents a short version of the more extensive Borderline Symptom List (87) for which excellent psychometric properties were reported. Similar results were found for the short version used in this study (86).The French version has shown comparable validity coefficients (88). The Inventory of Interpersonal Problems (IIP, (89); French translation by Stigler) is a self-report questionnaire assessing interpersonal patterns on several dimensions, such as affirmation, affiliation, submission, intimacy, responsibility and control. In total, this questionnaire comprises 64 items. Spielberger State-Trait Anger Inventory (STAXI-2;(90)) is a self-report questionnaire on trait and state anger, using 44 items. The French validation and adaptation was carried out by Borteyrou, Bouchon-Schweitzer and Spielberger (91). The Difficulties in Emotion Regulation Scale (DERS; (92)) is a self-report questionnaire assessing the quality of emotion regulation using 36 items. The French translation and validation of this instrument yielded satisfactory factor structure on a student sample (93). As measure of the therapeutic alliance, the Working Alliance Inventory (WAl; (94); French validation (95)) will be given after each session; the therapeutic alliance will be introduced as moderator where appropriate. At intake, reliable psychiatric 
diagnoses (using SCID-5-CV and SCID-5-PD by American Psychiatric Association), childhood trauma (Childhood Trauma Questionnaire; (96); French version(97)), rejection sensitivity (Rejection Sensitivity Questionnaire-Adult; (98)) and the level of intelligence (NART reading test, French version (99)) will be assessed.

Manipulation checks will be introduced by using self-report questionnaires of arousal (Self-Assessment Manikin; SAM; (100)), self-esteem (State Self-Esteem Scale; SSES;(101)) and vividness (Vividness of Visual Imagery Questionnaire; VVIQ; $(102,103))$.

\section{Research Procedure}

After the patient makes a request for treatment at the Department of Psychiatry, University of Lausanne, for problems related with BPD, the patient meets with a researcher who explains to him/her the study and informs him/her about the randomization and the assessment schedule. Upon approval, patients are block-randomized (by 10) and attributed either to a) a 4-month GPM or b) 4-month TAU (see Fig. 1). Patients are paid CHF 70.- for full participation in a assessment point.

Data analytic plan

For the behavioral assessments, we will conduct intent-to-treat and completer analyses for all variables (hypothesis 1: outcome, defined as residual gains at discharge). We will use multilevel modeling (104) where appropriate, for hypotheses 2 and 3 (global change and treatment response). For hypothesis 4 (mediation), we will conduct a mediation analysis for both potential mechanisms of change (105). Raw and composite scores for outcome and all mechanisms of change indexes will be used, by controlling for the corresponding fMRI data from the same assessment point. Composite scores involve for EP combining pre-post change in contemptuousness with change in neuronal regions of interest for individualized self-criticism and for SCP combining pre-post change in CCRT pervasiveness with change in neuronal regions of interest for theory of mind.

Therapist effects will be controlled for in the three-level HLM (106). All indexes (i.e., behavioral and fMRI) of the patient groups $(\mathrm{N}=80)$ at intake will be rigorously compared with the indexes found for the healthy control $(\mathrm{N}=20)$; we expect systematic between-group differences, in the context of a control analysis. Statistical treatment packages HLM7 and SPSS23 will be used for the analyses of the behavioral indexes.

For the $\mathrm{fMRI}$ assessments, we will use the methodology of blood-oxygen-level-dependant (BOLD) imaging followed by standard data processing and statistical analysis in the framework of SPM12. The fMRI data will be acquired on a Siemens Prisma 3T (64-channel head coil using a 2D EPI sequence). The acquisition parameters will be as follows: $3 \times 3 \times 3 \mathrm{~mm}$ : TE $=30 \mathrm{~ms}$, slice TR $=66 \mathrm{~ms}, 30$ slices, flip angle $=90^{\circ}$. The structural MRI data consists of T1-weighted MPRAGE images $(\mathrm{TR}=2000 \mathrm{~ms} ; \mathrm{TI}=$ $920 \mathrm{~ms} ; \alpha=9^{\circ} ; B W=250 \mathrm{~Hz}$ / pixel; readout in inferior-superior direction; FoV $=256 \times 232 \mathrm{~mm}$; 176 slices) at $1 \mathrm{~mm}$ resolution. All data pre-processing will be performed using the Statistical Parametric Mapping 
software (SPM12; Wellcome Trust Centre for Neuroimaging, shttp://www.fil.ion.ucl.ac.uk/spm/) running under Matlab 7.13 (The MathWorks, Inc., Natick, MA, USA). EPI images will be realigned to the subject's average image across trials (corrected for spatial distortions using the SPM fieldmap tools). The parameters of registration to standardized MNI space will be calculated on the anatomical image and the default settings of the "unified segmentation" framework followed by the diffeomorphic registration algorithm DARTEL $(107,108)$. The spatial registration parameters will be applied to the functional timeseries co-registered to the corresponding individual's anatomical scan. Prior to statistical analysis, we will apply a spatial smoothing with a Gaussian kernel of $8 \mathrm{~mm}$ full-width-at-half-maximum. All statistical analyses will be performed using the default settings in SPM12. The statistical analysis at subjectspecific level will be performed using the General Linear Model (GLM) after convolving the event onsets with a canonical hemodynamic response function (109). Both time-points will be modelled as two separate sessions within the design matrices. For the EP task, we will calculate at the subject level the interaction between WORDS (self-critical vs standard negative words; the non-words and the negative words will be excluded from the analyses, but used as control variables to ensure cognitive appropriateness) and TIME (time point 1 vs time point 2) using symbols as baseline. For the SCP task, the subject-level differential t-contrast will test the interaction between ToM, PUN and time point (the control stimuli will be excluded from the data analysis, but will serve as control for cognitive appropriateness). For both tasks, we will use a one-sample t-test along with the outcomes and arousal changes associated with treatment as regressors for the group-level analyses. The differential contrasts at the group level will test the positive and negative correlation between the interaction at the subjectspecific level and BOLD signal changes. Where appropriate, we will control for the corresponding behavioural data from the same assessment point.

Monitoring and ethics

According to the accepted Data Management Plan, data will be entered into RedCap on a secured space on the University server. This program allows full accountability of data management, and should problems arise, standard procedures are in place. As such, data safety is guaranteed by the system's security check and no formal data monitoring committee is requested (confidentiality). The competent Ethics Committee can request audits at any moment in time and the primary investigator and his team will follow its instructions. Audit may include to disclose, in a restricted fashion and only if deemed necessary, personal data related to participants to the Ethics Committee. The same Data Management Plan outlines procedures in case of adverse events in the context of the trial which includes provision, if needed of post-trial care in case of harm .

The trial was approved by the competent ethics committee (see above) and potential amendments will have to be approved by the same, and be communicated to publishing journals. Informed consent will be obtained before the inclusion process can start by the competent researcher. For the fMRI part, this procedure involves the complete information on the safety recommendations in this environment. Participants' personal information will be stored at a separate, locked, place at the Department of Psychiatry. No personal information will be revealed neither before, during nor after the trial (except for 
the case of audit by the competent Ethics Committee; confidentiality ; plans to give access to protocol, participant level-data and statistical code are described in the Data Management Plan ).

Publications of the results will be encouraged to all relevant groups (i.e., scientific publication, communication at conferences, communication with stakeholders, patients and families, ).

Feasibility: Results From The Pilot Study

We demonstrated feasibility of the pre-post design (50). We demonstrated for $\mathrm{N}=8$ medication-free righthanded female patients with BPD undergoing a 10-session psychiatric treatment that hypotheses 1 and 2 may be confirmed (due to the small sample size, the analyses for hypotheses 3 and 4 were not tested). The behavioral pre-post treatment outcome effect sizes ranged between $d=0.41$ (for OQ-45) and $d=0.51$ (for BSL-23). We observed arousal increase within session of the two-chair dialogue $(d=0.36)$, paralleled by a large decrease in peak arousal between pre- and post-treatment $(d=0.80)$. In the EP task, we demonstrated treatment associated trends for neural activity reduction in the associative parts of putamen when exposed to the individual's own self-critical words. The exposure to ToM stimuli revealed trends for treatment related neural activity modulation in the OFC, ACC and NAcC, and the medio-dorsal nucleus of the thalamus. Neural activity (i.e., in the precuneus, left amygdala) related with the behavioral changes in arousal, but remained independent from outcome, whereas change in arousal was related with symptom reduction. Feasibility of the trial and relevance of the pre-post hypotheses are therefore demonstrated.

\section{Discussion}

Borderline Personality Disorder is among the most debilitating and lethal mental disorders. Each year, millions are spent on direct and indirect costs related to this disorder and thousands of patients complete suicide, profoundly impacting the lives of those left behind. Effective treatments of BPD exist, but remain difficult to disseminate. Brief psychiatric treatment is cost-effective and may produce, to some extend, similar effects than a structured psychotherapy program, at least may represent a promising initial treatment in a stepped-care approach: we think that its implementation should therefore be a major priority in the health care system. This is the first study testing the effectiveness of such brief guidelinebased psychiatric treatment (compared to a treatment as usual) and its underlying mechanisms of change. The latter will be done by taking into account the individual's subjective perspective in the assessment. The integrated methodology optimally compensates for respective limitations of psychotherapy process and neurofunctional assessments, making it the scientifically most precise, and the clinically and ecologically most relevant approach, to the measurement of mechanisms of change in treatment research. Therefore, this study does not only contribute to the understanding of the effects of treatment as a whole, but also should help render even more effective treatments for BPD by a clinically relevant understanding of how change is produced through psychological treatment.

\section{Trial Status}


This study is currently ongoing and is not completed (protocol version number 2 from February 9th, 2018; start of recruitment December 1st, 2018; projected end of recruitment August 31st, 2021).

\section{Declarations}

\section{Acknowledgments}

We thank the collaborating researchers and clinicians for their important contribution to this trial.

\section{Authors' contributions \{31b\}}

All co-authors of this protocol have collaborated in the elaboration, with the first author (UK) being responsible for the overall scientific quality, patient recruitment and monitoring, as well as scientific head of the study.

\section{Funding $\{4\}$}

This trial is supported by the Swiss National Science Foundation (SNSF100014_179457/1, to Dr. Kramer). The study was designed independently from the funding source, as the data collection, analysis and interpretation is not influenced by the funding source, in keeping with the regulations of SNSF.

\section{Availability of data and materials $\{29\}$}

All co-authors of this of this protocol will have full access to the data. Restrictions to this principle are described in an internal document. The datasets analyzed during the current study are available from the corresponding author on reasonable request and pending ethical approval.

\section{Ethics approval and consent to participate $\{24\}$ and consent for publication $\{32\}$}

With respect to the submitted project on the SwissEthics plateform, the competent Canton de Vaud Ethics Committee, has approved the study (2017-02167).

\section{Competing interests}

The authors declare that they have no competing interests.

\section{References}

1. Bender DS, Dolan RT, Skodol AE, Sanislow CA, Dyck IR, McGlashan TH, et al. Treatment utilization by patients with personality disorders. American Journal of Psychiatry 2001;158(2):295-302.

2. Soeteman DI, Roijen LH-v, Verheul R, Busschbach JJ. The economic burden of personality disorders in mental health care. Journal of Clinical Psychiatry 2008;69(2):259.

3. Association AP. Practice guideline for the treatment of patients with borderline personality disorder: American Psychiatric Pub; 2001. 
4. Budge SL, Moore JT, Del Re A, Wampold BE, Baardseth TP, Nienhuis JB. The effectiveness of evidence-based treatments for personality disorders when comparing treatment-as-usual and bona fide treatments. Clinical Psychology Review 2013;33(8):1057-66.

5. Cristea IA, Gentili C, Cotet CD, Palomba D, Barbui C, Cuijpers P. Efficacy of psychotherapies for borderline personality disorder: a systematic review and meta-analysis. JAMA Psychiatry 2017;74(4):319-28.

6. Herpertz SC, Rudolf, G. Lieb, K. . Borderline-Persönlickkeitsstörungen. In: Herpertz SC, Caspar F., Lieb K., editor. Psychotherapie Funktions- und störungsorientiertes Vorgehen. München: Elsevier; 2016. p. 395-412.

7. Clarkin J. Raising the bar in the empirical investigation of psychotherapy. American Psychiatric Association; 2014.

8. Clarkin JF, Levy KN. Psychotherapy for patients with borderline personality disorder: Focusing on the mechanisms of change. Journal of Clinical Psychology 2006;62(4):405-10.

9. Forster C, Berthollier N, Rawlinson D. A systematic review of potential mechanisms of change in psychotherapeutic interventions for personality disorder. Journal of Psychology and Psychotherapy. 2014;4(133):2161-0487.1000133.

10. Johansson P, Høglend P. Identifying mechanisms of change in psychotherapy: Mediators of treatment outcome. Clinical Psychology and Psychotherapy 2007;14(1):1-9.

11. Kazdin A. Understanding how and why psychotherapy leads to change. Psychotherapy Research 2009;19(4-5):418-28.

12. Kramer U. Case formulation for personality disorder: Tailoring psychotherapy to the individual client. Cambridge, MA: Elsevier; 2019.

13. Rudge S, Feigenbaum JD, Fonagy P. Mechanisms of change in dialectical behaviour therapy and cognitive behaviour therapy for borderline personality disorder: a critical review of the literature. Journal of Mental Health 2017:1-11.

14. Euler S, Dammann G, Endtner K, Leihener F, Perroud NA, Reisch T, et al. Borderline-Störung: Behandlungsempfehlungen der SGPP. 2018;169(05):135-43.

15. Choi-Kain LW, Finch EF, Masland SR, Jenkins JA, Unruh BT. What works in the treatment of borderline personality disorder. Current Behavior and Neuroscience Reports. 2017;4(1):21-30.

16. Gunderson JG, Stout RL, McGlashan TH, Shea MT, Morey LC, Grilo CM, et al. Ten-year course of borderline personality disorder: psychopathology and function from the Collaborative Longitudinal Personality Disorders study. Archives of general psychiatry 2011;68(8):827-37.

17. McMain SF, Guimond T, Streiner DL, Cardish RJ, Links PS. Dialectical behavior therapy compared with general psychiatric management for borderline personality disorder: clinical outcomes and functioning over a 2-year follow-up. American Journal of Psychiatry 2012;169(6):650-61.

18. McMain SF, Links PS, Gnam WH, Guimond T, Cardish RJ, Korman L, et al. A randomized trial of dialectical behavior therapy versus general psychiatric management for borderline personality disorder. American Journal of Psychiatry. 2009;166(12):1365-74. 
19. Gunderson JG, Links PS. Borderline personality disorder: A clinical guide. Washington DC: American Psychiatric Publishing; 2008. 1-28 p.

20. Gunderson JG, Links PS. Handbook of good psychiatric management for borderline personality disorder: American Psychiatric Publishing Inc.; 2014.

21. Bateman A, Fonagy P. 8-year follow-up of patients treated for borderline personality disorder: mentalization-based treatment versus treatment as usual. American Journal of Psychiatry 2008;165(5):631-8.

22. Gunderson JG. The emergence of a generalist model to meet public health needs for patients with borderline personality disorder. American Journal of Psychiatry 2016;173(5):452-8.

23. Kramer U, Charbon P, Despland J-N, Kolly S. "Good enough" training in clinical practice for BPD? Swiss Archives of Neurology, Psychiatry and Psychotherapy 2017;168(8).

24. Chanen AM, Berk M, Thompson K. Integrating early intervention for borderline personality disorder and mood disorders. Harvard Review of psychiatry 2016;24(5):330-41.

25. Choi-Kain LW, Albert EB, Gunderson JG. Evidence-based treatments for borderline personality disorder: implementation, integration, and stepped care. Harvard Review of Psychiatry 2016;24(5):342-56.

26. Paris J. Stepped care and rehabilitation for patients recovering from borderline personality disorder. Journal of Clinical Psychology: In Session 2015;71(8):747-52.

27. Berthoud L, Pascual-Leone A, Caspar F, Tissot H, Keller S, Rohde KB, et al. Leaving distress behind: A randomized controlled study on change in emotional processing in borderline personality disorder. Psychiatry: Interpersonal and Biological Processes. 2017;80(2):139-54.

28. Kramer U, Keller S, Caspar F, de Roten Y, Despland J-N, Kolly S. Early change in coping strategies in responsive treatments for borderline personality disorder: A mediation analysis. Journal of Consulting and Clinical Psychology 2017;85(5):530.

29. Levy KN, Meehan KB, Kelly KM, Reynoso JS, Weber M, Clarkin JF, et al. Change in attachment patterns and reflective function in a randomized control trial of transference-focused psychotherapy for borderline personality disorder. Journal of Consulting and Clinical Psychology 2006;74(6):1027.

30. Kramer U, Stiles WB. The responsiveness problem in psychotherapy: A review of proposed solutions. Clinical Psychology: Science and Practice 2015;22(3):277-95.

31. Schnell K, Herpertz SC. Effects of dialectic-behavioral-therapy on the neural correlates of affective hyperarousal in borderline personality disorder. Journal of Psychiatric Research 2007;41(10):837-47.

32. Schmitt R, Winter D, Niedtfeld I, Herpertz SC, Schmahl CJBPCN. Effects of psychotherapy on neuronal correlates of reappraisal in female patients with borderline personality disorder. Biological Psychiatry: Cognitive Neuroscience \& Neuroimaging. 2016;1(6):548-57.

33. Pascual-Leone A, Herpertz SC, Kramer U. Experimental designs and the 'emotion stimulus critique': Hidden problems and potential solutions in the study of emotion. Psychopathology 2016;49(1):60-8. 
34. Kramer U, Pascual-Leone A, Despland J-N, de Roten Y. One minute of grief: Emotional processing in short-term dynamic psychotherapy for adjustment disorder. Journal of Consulting and Clinical Psychology 2015;83(1):187.

35. Kramer U, Pascual-Leone A, Rohde KB, Sachse R. Emotional processing, interaction process, and outcome in clarification-oriented psychotherapy for personality disorders: A process-outcome analysis. Journal of Personality Disorders 2016;30(3):373-94.

36. Tashiro T, Mortensen L. Translational research: How social psychology can improve psychotherapy. American Psychologist 2006;61(9):959.

37. McMain S, Pos A, Iwakabe S. Facilitating emotion regulation: General principles for psychotherapy. Psychotherapy Bulletin. 2010;45(3):16-21.

38. Greenberg LS, Pascual-Leone A. Emotion in psychotherapy: A practice-friendly research review. Journal of Clinical Psychology: In Session 2006;62(5):611-30.

39. Neacsiu AD, Rizvi SL, Linehan MMJBr. Dialectical behavior therapy skills use as a mediator and outcome of treatment for borderline personality disorder. Behaviour Research and Therapy 2010;48(9):832-9.

40. Schulze L, Domes G, Krüger A, Berger C, Fleischer M, Prehn K, et al. Neuronal correlates of cognitive reappraisal in borderline patients with affective instability. Biological Psychiatry 2011;69(6):564-73.

41. New AS, Hazlett EA, Buchsbaum MS, Goodman M, Mitelman SA, Newmark R, et al. Amygdalaprefrontal disconnection in borderline personality disorder. Neuropsychopharmacology 2007;32(7):1629.

42. Goodman M, Carpenter D, Tang CY, Goldstein KE, Avedon J, Fernandez N, et al. Dialectical behavior therapy alters emotion regulation and amygdala activity in patients with borderline personality disorder. Journal of Psychiatric Research 2014;57:108-16.

43. Goodman $M$, editor Changes in amygdala activation and connectivity in DBT treatment response in BPD. North America Society for the Study of Personality Disorders (NASSPD) Conference; 2016; New York.

44. Winter D, Niedtfeld I, Schmitt R, Bohus M, Schmahl C, Herpertz SC. Neural correlates of distraction in borderline personality disorder before and after dialectical behavior therapy. European Archives of Psychiatry and Clinical Neuroscience. 2017;267(1):51-62.

45. Niedtfeld I, Schmitt R, Winter D, Bohus M, Schmahl C, Herpertz SC. Pain-mediated affect regulation is reduced after dialectical behavior therapy in borderline personality disorder: a longitudinal fMRI study. Social Cognitive and Affective Neuroscience. 2017;12(5):739-47.

46. Perez DL, Vago DR, Pan H, Root J, Tuescher O, Fuchs BH, et al. Frontolimbic neural circuit changes in emotional processing and inhibitory control associated with clinical improvement following transference-focused psychotherapy in borderline personality disorder. Psychiatry and Clinical Neurosciences. 2016;70(1):51-61.

47. Pascual-Leone A. Dynamic emotional processing in experiential therapy: Two steps forward, one step back. Journal of Consulting and Clinical Psychology. 2009;77(1):113. 
48. Kramer U, Pascual-Leone A. The role of maladaptive anger in self-criticism: A quasi-experimental study on emotional processes. Counselling Psychology Quarterly 2016;29(3):311-33.

49. Kramer U, Pascual-Leone A, Berthoud L, De Roten Y, Marquet P, Kolly S, et al. Assertive anger mediates effects of dialectical behaviour-informed skills training for borderline personality disorder: A randomized controlled trial. Clinical psychology and Psychotherapy. 2016;23(3):189-202.

50. Kramer U, Kolly S, Maillard P, Pascual-Leone A, Samson AC, Schmitt R, et al. Change in Emotional and Theory of Mind Processing in Borderline Personality Disorder: A Pilot Study. The Journal of Nervous and Mental Disease. 2018;206(12):935-43.

51. Kramer U, Pascual-Leone A. Self-knowledge in personality disorders: An emotion-focused perspective. Journal of Personality Disorders. 2018;32(3):329-50.

52. Fertuk E, editor Rejection sensitivity predicts cortisol reactivity in borderline peronality disorder and depressive disorders. North American Society for the Study of Personality Disorders (NASSPD) Conference; 2016; New York

53. Luyten P, Lowyck B, Blatt SJ. Mechanisms of change through the lens of two-polarities models of personality development: State of the art and new directions. Psychoanalytic Inquiry. 2017;37(3):17990.

54. Pos $A E$, Greenberg LS. Organizing awareness and increasing emotion regulation: Revising chair work in emotion-focused therapy for borderline personality disorder. Journal of Personality Disorders. 2012;26(1):84-107.

55. Whelton WJ, Greenberg LS. Emotion in self-criticism. Personality and Individual Differences. 2005;38(7):1583-95.

56. Longe O, Maratos FA, Gilbert P, Evans G, Volker F, Rockliff H, et al. Having a word with yourself: Neural correlates of self-criticism and self-reassurance. Neurolmage. 2010;49(2):1849-56.

57. Davidson RJ, Pizzagalli D, Nitschke JB, Putnam K. Depression: perspectives from affective neuroscience. Annual Review of Psychology. 2002;53(1):545-74.

58. Calder AJ, Lawrence AD, Young AW. Neuropsychology of fear and loathing. Nature Reviews Neuroscience. 2001;2(5):352.

59. Doerig N, Schlumpf Y, Spinelli S, Späti J, Brakowski J, Quednow BB, et al. Neural representation and clinically relevant moderators of individualised self-criticism in healthy subjects. Social Cognitive and Affective Neuroscience. 2013;9(9):1333-40.

60. Choi-Kain LW, Gunderson JG. Mentalization: Ontogeny, assessment, and application in the treatment of borderline personality disorder. American Journal of Psychiatry. 2008;165(9):1127-35.

61. Fonagy P, Luyten P, Bateman A. Translation: Mentalizing as treatment target in borderline personality disorder. Personality Disorders: Theory, Research, and Treatment. 2015;6(4):380.

62. Fischer-Kern M, Doering S, Taubner S, Hörz S, Zimmermann J, Rentrop M, et al. Transference-focused psychotherapy for borderline personality disorder: Change in reflective function. The British Journal of Psychiatry. 2015;207(2):173-4. 
63. De Meulemeester C, Vansteelandt K, Luyten P, Lowyck B. Mentalizing as a mechanism of change in the treatment of patients with borderline personality disorder: A parallel process growth modeling approach. Personality Disorders: Theory, Research, and Treatment. 2018;9(1):22.

64. Maillard P, Dimaggio G, de Roten Y, Berthoud L, Despland J-N, Kramer U. Metacognition as a predictor of change in the treatment for borderline personality disorder: A preliminary pilot study. Journal of Psychotherapy Integration. 2017;27(4):445.

65. Antonsen BT, Johansen MS, Rø FG, Kvarstein EH, Wilberg T. Is reflective functioning associated with clinical symptoms and long-term course in patients with personality disorders? Comprehensive Psychiatry. 2016;64:46-58.

66. Gullestad FS, Johansen MS, Høglend P, Karterud S, Wilberg T. Mentalization as a moderator of treatment effects: Findings from a randomized clinical trial for personality disorders. Psychotherapy Research. 2013;23(6):674-89.

67. Luborsky L, Crits-Christoph P. Understanding transference: The core conflictual relationship theme method: American Psychological Association; 1998.

68. Drapeau M, Perry JC. The core conflictual relationship themes (CCRT) in borderline personality disorder. Journal of Personality Disorders. 2009;23(4):425-31.

69. Drapeau M, Perry JC, Körner A. Interpersonal patterns in borderline personality disorder. Journal of personality disorders. 2012;26(4):583-92.

70. O'Neill A, D’Souza A, Samson AC, Carballedo A, Kerskens C, Frodl T. Dysregulation between emotion and theory of mind networks in borderline personality disorder. Psychiatry Research: Neuroimaging. 2015;231(1):25-32.

71. Schurz M, Radua J, Aichhorn M, Richlan F, Perner J. Fractionating theory of mind: a meta-analysis of functional brain imaging studies. Neuroscience \& Biobehavioral Reviews. 2014;42:9-34.

72. Spreng RN, Mar RA, Kim AS. The common neural basis of autobiographical memory, prospection, navigation, theory of mind, and the default mode: a quantitative meta-analysis. Journal of Cognitive Neuroscience. 2009;21(3):489-510.

73. Association AP. Diagnostic and Statistical Manual of Mental Disorders. BMC Med. 2013;17:133-7.

74. Keuroghlian AS, Palmer BA, Choi-Kain LW, Borba CP, Links PS, Gunderson JG. The effect of attending good psychiatric management (GPM) workshops on attitudes toward patients with borderline personality disorder. Journal of Personality Disorders. 2016;30(4):567-76.

75. Charbon P, Kramer U, Droz J, Kolly S. Implementation of good psychiatric management in 10 sessions. Applications of Good Psychiatric Management for Borderline Personality Disorder: A Practical Guide. 2019:233-52.

76. Kolly S, Charbon P, Kramer, U. Trouble de la personnalité borderline-Pratiques thérapeutiques: Elsevier Health Sciences; 2019.

77. Gunderson JG, Lyons-Ruth K. BPD's interpersonal hypersensitivity phenotype: A gene-environmentdevelopmental model. Journal of Personality Disorders. 2008;22(1):22-41. 
78. Zanarini MC. Zanarini Rating Scale for Borderline Personality Disorder (ZAN-BPD): a continuous measure of DSM-IV borderline psychopathology. Journal of Personality Disorders. 2003;17(3):23342.

79. Greenberg LS. Emotion-focused therapy: Coaching clients to work through their feelings: American Psychological Association; 2015.

80. Pascual-Leone A, Greenberg LS. Classification of Affective-Meaning-States (CAMS). Emotional processing in the therapeutic hour: "Why the only way out is through" 2005.

81. Kherif F, Josse G, Price CJ. Automatic top-down processing explains common left occipito-temporal responses to visual words and objects. Cerebral Cortex. 2010;21(1):103-14.

82. Samson AC, Zysset S, Huber O. Cognitive humor processing: different logical mechanisms in nonverbal cartoons-an fMRI study. Social Neuroscience. 2008;3(2):125-40.

83. Samson AC, Hempelmann CF, Huber O, Zysset S. Neural substrates of incongruity-resolution and nonsense humor. Neuropsychologia. 2009;47(4):1023-33.

84. Lambert M, Morton J, Hatfield D, Harmon C, Hamilton S, Reid R, et al. Administration and scoring manual for the OQ-45.2 (Outcome Questionnaire). Orem, UT: American Professional Credentialing Services. 2004.

85. Emond C, Savard K, Lalande G, Boisvert N, Boutin M, Simard V, editors. Propriétés psychométriques de la Mesure de l'Impact (MI-45), version francophone du Outcome Questionnaire-45 (OQ-45.2) [Psychometric characteristics of the 0Q-45, French Version]. American College of Foot and Ankle Surgeons conference, Montreal, QC; 2004.

86. Bohus M, Kleindienst N, Limberger MF, Stieglitz R-D, Domsalla M, Chapman AL, et al. The short version of the Borderline Symptom List (BSL-23): development and initial data on psychometric properties. Psychopathology. 2009;42(1):32-9.

87. Bohus M, Limberger MF, Frank U, Chapman AL, Kühler T, Stieglitz R-D. Psychometric properties of the borderline symptom list (BSL). Psychopathology. 2007;40(2):126-32.

88. Nicastro R, Prada P, Kung A-L, Salamin V, Dayer A, Aubry J-M, et al. Psychometric properties of the French borderline symptom list, short form (BSL-23). Borderline Personality Disorder and Emotion Dysregulation. 2016;3(1):4.

89. Horowitz LM, Rosenberg SE, Baer BA, Ureño G, Villaseñor VS. Inventory of interpersonal problems: psychometric properties and clinical applications. Journal of Consulting and Clinical Psychology. 1988;56(6):885.

90. Spielberger CD. Professional manual for the state-trait anger expression inventory (STAXI). Odessa, FL: Psychological Assessment Resources 1988.

91. Borteyrou X, Bruchon-Schweitzer M, Spielberger C. Une adaptation française du STAXI-2, inventaire de colère-trait et de colère-état de CD Spielberger. L'Encéphale. 2008;34(3):249-55.

92. Gratz KL, Roemer L. Multidimensional assessment of emotion regulation and dysregulation: Development, factor structure, and initial validation of the difficulties in emotion regulation scale. Journal of Psychopathology and Behavioral Assessment. 2004;26(1):41-54. 
93. Dan-Glauser ES, Scherer KR. The difficulties in emotion regulation scale (DERS). Swiss Journal of Psychology. 2012.

94. Horvath AO, Greenberg LS. Development and validation of the Working Alliance Inventory. Journal of Counseling Psychology. 1989;36(2):223.

95. Corbière M, Bisson J, Lauzon S, Ricard N. Factorial validation of a French short-form of the Working Alliance Inventory. International Journal of Methods in Psychiatric Research. 2006;15(1):36-45.

96. Bernstein DP, Stein JA, Newcomb MD, Walker E, Pogge D, Ahluvalia T, et al. Development and validation of a brief screening version of the Childhood Trauma Questionnaire. Child Abuse \& Neglect. 2003;27(2):169-90.

97. Paquette D, Laporte L, Bigras M, Zoccolillo M. Validation de la version française du CTQ et prévalence de l'histoire de maltraitance. Santé mentale au Québec. 2004;29(1):201-20.

98. Downey G, Feldman SI. Implications of rejection sensitivity for intimate relationships. Journal of Personality and Social Psychology. 1996;70(6):1327.

99. Mackinnon A, Mulligan R. The estimation of premorbid intelligence levels in French speakers. L'Encéphale. 2005;31(1 Pt 1):31-43.

100. Bradley MM, Lang PJ. Measuring emotion: the self-assessment manikin and the semantic differential. Journal of Behavior Therapy and Experimental Psychiatry. 1994;25(1):49-59.

101. Heatherton TF, Polivy J. Development and validation of a scale for measuring state self-esteem. Journal of Personality and Social psychology. 1991;60(6):895.

102. Marks DF. Visual imagery differences in the recall of pictures. British journal of Psychology. 1973;64(1):17-24.

103. McKelvie S. The VVIQ as a psychometric test of individual differences in visual imagery vividness: $A$ critical quantitative review and plea for direction. Journal of Mental Imagery. 1995;19:1-106.

104. Bryk AS, Raudenbush SW. Application of hierarchical linear models to assessing change. Psychological Bulletin. 1987;101(1):147.

105. Hayes AF. Partial, conditional, and moderated moderated mediation: Quantification, inference, and interpretation. Communication Monographs. 2018;85(1):4-40.

106. Baldwin SA, Imel Z. Therapist effects: Findings and methods. Bergin and Garfield's handbook of psychotherapy and behavior change. 2013;6:258-97.

107. Ashburner J, Friston KJ. Unified segmentation. Neuroimage. 2005;26(3):839-51.

108. Ashburner J. A fast diffeomorphic image registration algorithm. Neuroimage. 2007;38(1):95-113.

109. Worsley KJ, Friston KJ. Analysis of fMRI time-series revisited-again. Neuroimage. 1995;2(3):173-81.

\section{Figures}




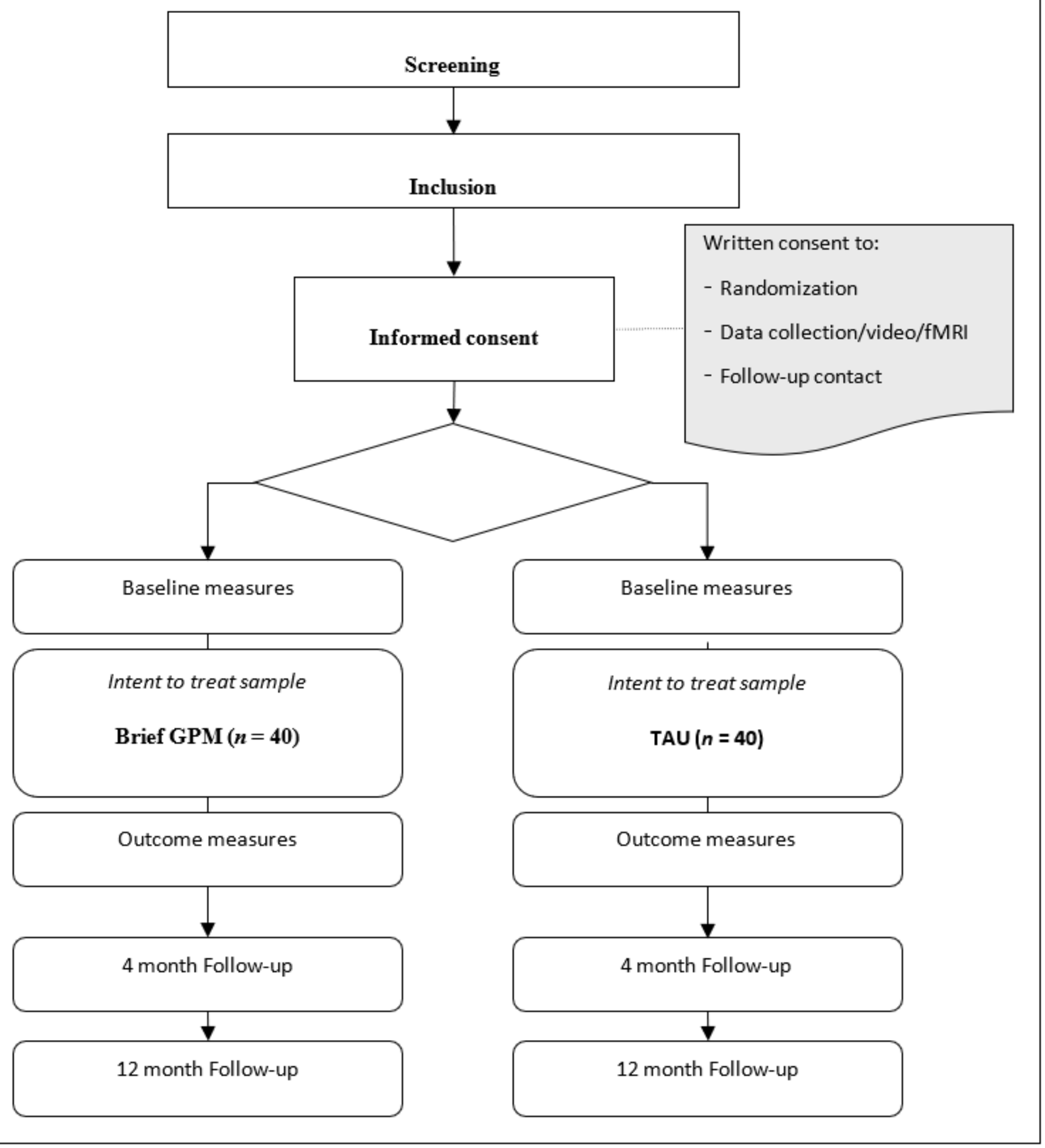

Figure 1

CONSORT flowchart of the research procedure

\section{Supplementary Files}

This is a list of supplementary files associated with this preprint. Click to download. 
- DecisionSNSFKramer2018.pdf

- CONSORT2010ChecklistKramerPopulated.doc

- translationsCERVDSNSF.docx

- renamed848a0.pdf 